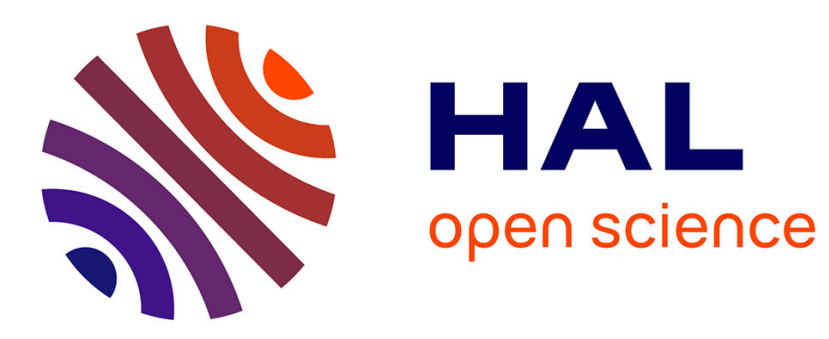

\title{
A model of influence with a continuum of actions
}

\author{
Michel Grabisch, Agnieszka Rusinowska
}

\section{To cite this version:}

Michel Grabisch, Agnieszka Rusinowska. A model of influence with a continuum of actions. Journal of Mathematical Economics, 2011, 47, pp.576-587. 10.1016/j.jmateco.2011.08.004 . hal-00666821

\section{HAL Id: hal-00666821 \\ https://hal.science/hal-00666821}

Submitted on 6 Feb 2012

HAL is a multi-disciplinary open access archive for the deposit and dissemination of scientific research documents, whether they are published or not. The documents may come from teaching and research institutions in France or abroad, or from public or private research centers.
L'archive ouverte pluridisciplinaire HAL, est destinée au dépôt et à la diffusion de documents scientifiques de niveau recherche, publiés ou non, émanant des établissements d'enseignement et de recherche français ou étrangers, des laboratoires publics ou privés. 


\title{
A model of influence with a continuum of actions ${ }^{\star}$
}

\author{
MICHEL GRABISCH and AGNIESZKA RUSINOWSKA \\ Université Paris I Panthéon-Sorbonne, Centre d'Economie de la Sorbonne \\ 106-112 Bd de l'Hôpital, 75647 Paris Cedex 13, France \\ Phone: 0033144078 285, 0033144078 212, Fax: 0033144078301 \\ michel.grabisch@univ-paris1.fr \\ agnieszka.rusinowska@univ-paris1.fr
}

\begin{abstract}
We generalize a two-action (yes-no) model of influence to a framework in which every player has a continuum of actions, among which he has to choose one. We assume the set of actions to be an interval. Each player has an inclination to choose one of the actions. Due to influence among players, the final decision of a player, i.e., his choice of one action, may be different from his original inclination. In particular, a coalition of players with the same inclination may influence another player with different inclination, and as a result of this influence, the decision of the player is closer to the inclination of the influencing coalition than his inclination was. We introduce a measure of such a positive influence of a coalition on a player. Several unanimous influence functions in this generalized framework are considered. Also the set of fixed points under a given influence function is analyzed. Furthermore, we study linear influence functions and discuss their convergence. For a linear unanimous function, we find necessary and sufficient conditions for the existence of the positive influence of a coalition on a player, and we calculate the value of the influence index. We also introduce a measure of a negative influence of a coalition on a player.
\end{abstract}

\section{JEL Classification: C7, D7}

Keywords: action, decision, influence index, unanimous influence function, fixed point, linear influence function

Corresponding author: Agnieszka Rusinowska

\section{Introduction}

In the voting literature, usually binary voting is assumed, for instance, casting 'yes' or 'no' vote on a particular proposal or voting for one of two available candidates. However, one may find several works that extend the number of options in voting situations. For instance, voting systems with abstention (as a third option together with 'yes' and 'no' votes) are studied e.g. in Braham and Steffen (2002, [12]), Felsenthal and Machover (1997, 1998, 2001, [20-22]), Fishburn (1973, [23]). Works on voting systems with several levels of approval in the input and output can be found in Freixas (2005a, 2005b, [24, 25]), Freixas and Zwicker (2003, [26]). In particular, standard simple games and games with abstention can be easily defined in such an extended framework. Also in Hsiao and Raghavan (1993, [34]) multi-choice games are studied, and in Bolger (1986, 1993, 2000, 2002, [5-8]) games with $n$ players and $r$ alternatives are analyzed.

Considering a continuum of alternatives or choices in models of game theory and social choice theory is interesting both from a theoretical and an application point of view, since

\footnotetext{
* This research project is supported by the National Agency for Research (Agence Nationale de la Recherche), Reference: ANR-09-BLAN-0321-01. We like to thank cordially the anonymous referee, Philippe Bich, and René van den Brink for their useful remarks and suggestions.
} 
many real life situations can be modeled in the framework with a continuum of actions or options. However, not that many works so far, in particular in the voting literature, concern models with a continuum of alternatives. One of such examples is presented in Chang and Stauber (2006, [13]), where the authors extend a costly voting model with binary voting to a model in which a choice has to be made from a continuum of alternatives. The agents have to decide whether or not to participate in a collective decision-making process that determines the level of a public good. There are costs associated with the act of voting and the implementation of the voting outcome. In the social choice literature, models with a continuum of alternatives are studied, e.g., in Abdou (1988, [1]), Huang (2004, 2009, [35, 36]).

One of the concepts naturally related to voting situations is the concept of influence. Since social networks play a crucial role in the opinion formation, using the network approach to influence is of particular relevance. Several models related to influence in sociology and economics are linear, where agents' interaction patterns are described by a stochastic matrix. The pioneering work of this kind is due to DeGroot (1974, [18]). In his model, agents have their initial opinions on a subject. Each entry of the (stochastic) interaction matrix represents the weight that one agent places on the current belief of another agent in forming his belief for the next period. The beliefs are updated over time. In the literature, several studies and variations of the DeGroot model based on different updating rules, in particular, updating varying over time, are presented; see, e.g., Berger (1981, [4]), Friedkin and Johnsen (1990, 1997, [27, 28]), DeMarzo et al. (2003, [19]), Krause (2000, [39]), Lorenz (2005, [40]), Jackson (2008, [37]), Golub and Jackson (2010, [29]). A related model of influence is also presented in Asavathiratham (2000, [2]); see also Asavathiratham et al. (2001, [3]). His model consists of a network of nodes, each with a status evolving over time. The evolution of the status is according to an internal Markov chain, but transition probabilities depend not only on the current status of the node, but also on the statuses of the neighboring nodes. Among other works on interaction and influence, we like to mention the eigenvector-like notions of centrality and prestige by Katz (1953, [38]) and Bonacich (1987, [9]); see also Bonacich and Lloyd (2001, [10]), and a sociological model of interactions on networks by Conlisk (1976, [14]); see also Conlisk $(1978,1992,[15,16])$. Steffi Yang $(2009,[41])$ studies price stability of a capital market, where the dynamics of opinion formations is modeled by social networks. In particular, the author investigates the importance of a network structure in determining market stability. Cont and Löwe (2010, [17]) present a dynamic binary choice model in which pairwise influences are linked to a social distance between individuals. Agents' actions influence preferences of other agents and this influence depends on the similarity of agents' characteristics. The authors study dynamic equilibria of the model in the limit when the number of agents is large, examine the impact of the characteristics of an agent on his choice, and discuss the empirical content of the model.

The point of departure for the present paper is another framework of influence originally introduced in Hoede and Bakker (1982, [33]) and later studied, e.g., in Grabisch and Rusinowska (2010a, [30]). In the two-action model, each player has to make an acceptance-rejection decision, and he has an inclination to vote either 'yes' or 'no'. The inclination of a player is defined as a decision the player would make if he were to decide completely on his own. However, an agent may experience influence of other players, and as a consequence of such an influence, his decision may be different from his original in- 
clination. The influence between players is defined in a broad sense and may cover many possible situations: forcing by players that are higher in a hierarchical structure, following authorities, learning and observing the behavior of others, etc. The transformation of the inclinations into the decisions is represented by an influence function. In Grabisch and Rusinowska (2010a, [30]), we define and investigate weighted influence indices that measure influence between players in the yes-no model. We consider several special cases of the weighted influence indices and we analyze different kinds of influence. In Grabisch and Rusinowska (2010b, [31]) we enlarge the set of possible yes-no actions to a multi-action framework in which every player has a totally ordered set of possible actions, and he has an inclination to choose a particular action. We investigate the generalized influence indices and other tools related to the influence in the multi-choice model.

The present paper is a continuation of our research on the influence indices defined for the yes-no model and for the multi-action framework of influence. We consider now a generalization of the influence model in a social network and assume that each player has a continuum of actions to choose, and he has an inclination to choose one of them. Similar as in our former investigations on influence, we are interested in situations where a decision of a player (the final choice of one action) is different from his (preliminary) inclination. To be more precise, we define a positive influence index of a coalition on a player for the continuum case. This index measures how much the player chooses an action which is closer to the inclination of the coalition than his inclination was. We also define a negative influence index for the framework with a continuum of actions. The negative influence of a coalition on a player means that while the inclination of the player is different from the extreme action(s) of the coalition (that is, the action(s) lying as far as possible from the inclination of the coalition), the player's decision comes 'closer' to such an extreme action and goes farther from the inclination of the coalition. Furthermore, we define several unanimous influence functions for the continuum case, like the majority function, the guru function, the identity function, and the mass psychology function. Under such influence functions, unanimously inclined players always decide according to their common inclination. If this holds only for the extreme actions ( $\mathbf{0}$ and $\mathbf{1}$ for the model with the set of actions $[0,1]$ ), such an influence function is called unanimous on the boundaries. Moreover, we study the set of fixed points under a given influence function, i.e., the set of the inclination vectors that coincide with the decision vectors resulted from these inclinations. Fixed points are of fundamental interest in the analysis of the convergence of the influence process, since they remain invariant when iterating influence. Also linear influence functions, i.e., functions that can be written as a matrix, are studied. In particular, we discuss their convergence and the set of fixed points. For a function unanimous on the boundary 1 (for the set of actions $[0,1]$ ), we find necessary and sufficient conditions for the existence of a positive influence of a coalition on a player, and we calculate the value of the influence index. We also mention some related literature on linear influence models.

The structure of the paper is the following. In Section 2 we define the positive influence index for the model with a continuum of actions. In order to illustrate clearly the concepts introduced in the paper, in Section 3 we present examples of a two-agent social network and an analogous example of a three-player network. In Section 4 we investigate several unanimous influence functions. Sections 5 concerns fixed points of an influence function. 
In Section 6 we focus on linear influence functions. Section 7 is devoted to the concept of negative influence. In Section 8 we present some concluding remarks.

\section{The positive influence index in the generalized framework}

We consider a social network with the set of players (agents, actors) denoted by $N=$ $\{1, \ldots, n\}$. Each player has a continuum of actions to choose, i.e., we assume that the set of actions is an interval $[a, b]$, where $a, b \in \mathbb{R}$ and $a<b$. Each player has an inclination to choose one of the actions, i.e., by the inclination of a player we mean the particular action from $[a, b]$ the player wants to choose. Let $i_{m}$ denote the inclination of player $m \in N$, i.e., $i_{m} \in[a, b]$. Let $I=[a, b]^{n}$ denote the set of all $n$-inclination vectors ${ }^{1}$ $\mathbf{i}=\left(i_{1}, \ldots, i_{n}\right) \in[a, b]^{n}$.

It is assumed that players may influence each other, and due to the influence in the network, the final decision of a player may be different from his original inclination. In other words, each inclination vector $\mathbf{i} \in I$ is transformed into a decision vector $B(\mathbf{i})$, where $B: I \rightarrow I, \mathbf{i} \mapsto B(\mathbf{i})$ is the influence function. The decision vector $B(\mathbf{i})$ is an $n$-vector consisting of the decisions made by all the players, i.e., the actions chosen by the players. Our only requirement on the function $B$ is that it is measurable in Lebesgue's sense, i.e., the inverse image of any Borel set on $[a, b]^{n}$ is a Lebesgue measurable set. The set of all such influence functions with $N$ will be denoted by $\mathcal{B}$.

A coalition $\emptyset \neq S \subset N$ of players is assumed to be able to influence an outside agent $j \notin S$ only if all members of that coalition have the same inclination. In this case we say that the coalition is unanimous. Such $n$-inclination vectors where the coalition $S$ is unanimous form the set

$$
I_{S}:=\left\{\mathbf{i} \in[a, b]^{n}: \forall m, p \in S\left[i_{m}=i_{p}\right]\right\} .
$$

The cardinality of $S$ will be denoted by $s$. We use the notation $i_{S} \in[a, b]$ for the inclination of the members of the unanimous coalition $S$. This suggests to consider the whole coalition $S$ as a single "macro"-player, which we denote by $[S]$. Therefore, there is bijective correspondence between our $n$-dimensional vector $\mathbf{i} \in I_{S}$ and the $(n-s+1)$-dimensional vector $\mathbf{i}^{[S]} \in I_{S}^{[S]}$, defined by

$$
i_{j}^{[S]}:=i_{j} \text {, if } j \notin S, \text { and } i_{[S]}^{[S]}:=i_{S} .
$$

Let for each $\emptyset \neq S \subset N$ and $j \in N \backslash S$

$$
I_{S \rightarrow j}^{[S]}:=\left\{\mathbf{i}^{[S]} \in I_{S}^{[S]}: i_{j} \neq i_{S}\right\} .
$$

$I_{S \rightarrow j}^{[S]}$ denotes the set of all $(n-s+1)$-inclination vectors of potential positive influence of $S$ on $j$. These are all the $(n-s+1)$-inclination vectors for which the inclination of player $j$ is different from the inclination of coalition $S$.

\footnotetext{
${ }^{1}$ In the original model of influence introduced in Hoede and Bakker (1982, [33]) the inclination vectors $\mathbf{i}$ and decision vectors $B(\mathbf{i})$ are row vectors. For that reason and also for convenience, we keep the same convention in most of the sections of the present paper. However, in Section 6 on linear influence functions all the vectors are assumed to be column vectors.
} 
Next, for each $\emptyset \neq S \subset N, j \in N \backslash S$, and $B \in \mathcal{B}$, we define the set of all $(n-s+1)$ inclination vectors of (observed) positive influence of $S$ on $j$ under given $B$ as

$$
I_{S \rightarrow j}^{*[S]}(B)=\left\{\mathbf{i}^{[S]} \in I_{S \rightarrow j}^{[S]}:\left|(B(\mathbf{i}))_{j}-i_{S}\right|<\left|i_{j}-i_{S}\right|\right\},
$$

where it is understood that the vectors $\mathbf{i}^{[S]}$ and $\mathbf{i}$ in the above formula correspond to each other by $(2)$. The set $I_{S \rightarrow j}^{*[S]}(B)$ consists therefore of all the $(n-s+1)$-inclination vectors of potential positive influence of $S$ on $j$ such that the difference between the inclinations of $j$ and of $S$ is greater than the difference between the decision of player $j$ and the inclination of $S$. Consequently, we identify the cases where player $j$ chooses an action which is closer to the inclination of coalition $S$ than his inclination was.

Definition 1 Given $B \in \mathcal{B}$, for each $\emptyset \neq S \subset N, j \in N \backslash S$, the positive influence index of coalition $S$ on player $j$ under the influence function $B$ is defined as

$$
D(B, S \rightarrow j):=\frac{\int_{I_{S \rightarrow j}^{*[S]}(B)}\left[\left|i_{j}-i_{S}\right|-\left|(B(\mathbf{i}))_{j}-i_{S}\right|\right] d \lambda^{n-s+1}}{\int_{I_{S \rightarrow j}^{[S]}}\left|i_{j}-i_{S}\right| d \lambda^{n-s+1}},
$$

where the integral is understood in the Lebesgue sense, and $\lambda^{n-s+1}$ is the Lebesgue measure on $[a, b]^{n-s+1}$.

Note that due to the measurability assumption on $B$ and its boundedness, the Lebesgue integral in the numerator always exists, and since the denominator is non null, $D(B, S \rightarrow$ $j$ ) is well defined. We make some comments on its definition:

- $D(B, S \rightarrow j)$ is the proportion of situations where influence of $S$ on $j$ has been observed (the opinion of $j$ is closer to the one of $S$ after influence) among all situations where inclinations of $j$ and $S$ differ, this proportion being weighted by the intensity of influence (measured as the variation of distance between the opinions of $j$ and $S$ ). An important point is that we consider that influence of a coalition $S$ on an individual $j$ can occur only if the coalition $S$ is unanimous, i.e., all members have the same inclination. This restriction is very natural: if not all members of the coalition had the same opinion, a candidate for being influenced by that coalition would be doubting which player of the coalition to follow.

- This definition is a straightforward generalization of the influence index for the yes-no model (2010a, [30]) and for the model with a finite ordered set of actions (2010b, [31]).

- Observe that in the absence of influence ( $B$ is the identity function), we have $D(B, S \rightarrow$ $j)=0$. The maximum of influence of $S$ upon $j$ would occur if for any $\mathbf{i}$ in the domain, one has $B(\mathbf{i})_{j}=i_{S}$. In this case we obtain $D(B, S \rightarrow j)=1$, so clearly $D(B, S \rightarrow j) \in[0,1]$.

- Formula (5) can be simplified since the sets $I_{S}^{[S]}$ and $I_{S \rightarrow j}^{[S]}$ differ only by a set of measure 0 , namely the cylindrical extension of the diagonal $\left\{\mathbf{i}^{[S]}: i_{j}=i_{S}\right\}$. Therefore, the domains of integration can be taken as $I_{S}^{*[S]}(B)$ and $I_{S}^{[S]}$ in the numerator and denominator respectively, with $I_{S}^{*[S]}(B):=\left\{\mathbf{i}^{[S]} \in I_{S}^{[S]}: \mid\left(B(\mathbf{i})_{j}-i_{S}|<| i_{j}-i_{S} \mid\right\}\right.$.

- Although natural, our definition is not the only possible one: we use the $L_{1}$ norm to compute distances between opinions, inclinations, and also for the variation of distances. One could take as well other norms, typically $L_{2}$ which would lead to the 
Euclidean distance, or $L_{0}, L_{\infty}$, etc. We think however that the $L_{1}$ norm is the most appropriate since it is the most "linear" one. Using the $L_{2}$ norm would put too much emphasis on the large difference of opinions and large variations.

Lemma 1 If $I=[a, b]^{n}$, then for each $\emptyset \neq S \subset N$ and $j \in N \backslash S$

$$
\int_{I_{S \rightarrow j}^{[S]}}\left|i_{j}-i_{S}\right| d \lambda^{n-s+1}=\frac{(b-a)^{n-s+2}}{3} .
$$

\section{Proof:}

$$
\begin{aligned}
\int_{I_{S \rightarrow j}^{[S]}}\left|i_{j}-i_{S}\right| d \lambda^{n-s+1} & =2 \int_{a}^{b} \ldots \int_{a}^{b} \int_{a}^{b} \int_{a}^{i_{j}}\left(i_{j}-i_{S}\right) d \lambda_{S} d \lambda_{j} d \lambda_{k_{1}} \ldots d \lambda_{k_{n-s-1}} \\
& =2 \int_{a}^{b} \ldots \int_{a}^{b} \int_{a}^{b}\left[i_{j} \cdot i_{S}-\frac{i_{S}^{2}}{2}\right]_{i_{S}=a}^{i_{S}=i_{j}} d \lambda_{j} d \lambda_{k_{1}} \ldots d \lambda_{k_{n-s-1}} \\
& =\frac{1}{3} \int_{a}^{b} \ldots \int_{a}^{b}(b-a)^{3} d \lambda_{k_{1}} \ldots d \lambda_{k_{n-s-1}}=\frac{(b-a)^{n-s+2}}{3}
\end{aligned}
$$

\section{Examples}

\subsection{The two-player examples}

In order to illustrate the concepts introduced in Section 2, we present a very simple 2player example, i.e., $N=\{1,2\}$. We skip the upper index $[S]$, because for $n=2$ and all $\emptyset \neq S \subset N$, we have $N=[S] \cup(N \backslash S)$. Suppose that a two-member committee evaluates a scientific project and decides about the amount of funding for the project. Each of the two referees writes his report and proposes an amount of funding, i.e., chooses a percent (from $0 \%$ till $100 \%$ ) of the grant demanded by the project coordinator to be assigned to the project. Before preparing the reports, the referees discuss with each other the project. Each referee knows the inclination (opinion) of the another one, and such a pre-decision discussion is a good moment for exercising the influence between the referees.

We can assume that each player has to choose an action from the interval [0,1], where each action means a fraction of the demanded grant. In particular, the actions $0,0.5$ and 1 denote assigning no grant to the project, giving half of the requested grant, and giving the whole demanded amount, respectively. The set of inclination vectors is then equal to $I=[0,1]^{2}$. We consider the positive influence of player 1 on player 2 , and therefore

$$
I_{1 \rightarrow 2}=\left\{\left(i_{1}, i_{2}\right) \in[0,1]^{2}: i_{2} \neq i_{1}\right\} .
$$

These sets are presented in Figure 1 . The set of all inclination vectors $I$ consists of all the points $\left(i_{1}, i_{2}\right)$ of the square, while the set $I_{1 \rightarrow 2}$ consists of all the points of the square without the diagonal $i_{1}=i_{2}$. 

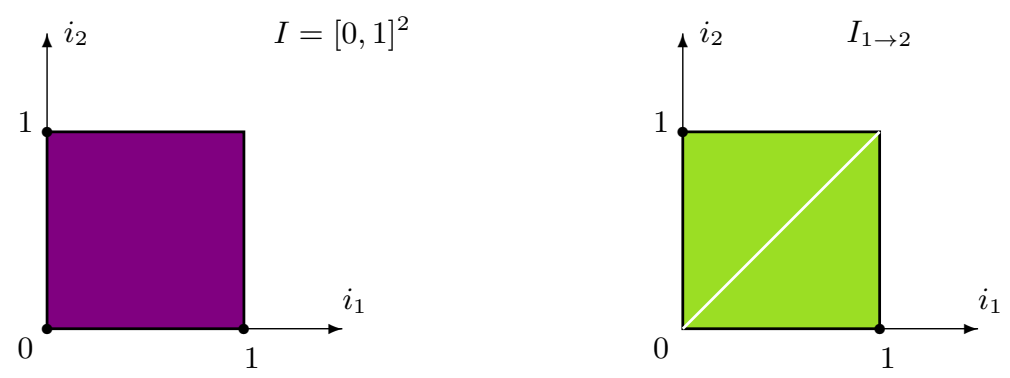

Fig. 1. The sets $I$ and $I_{1 \rightarrow 2}$ in the 2-player example

First, let us assume that the influence function $B$ is defined as follows:

$$
B\left(i_{1}, i_{2}\right)=\left(i_{1}, \frac{i_{1}+i_{2}}{2}\right) \text { for each }\left(i_{1}, i_{2}\right) \in[0,1]^{2},
$$

which means that player 1 always follows his own inclination, while the decision of player 2 is the middle point between the inclinations of the two players. Consequently, if their inclinations are not equal, then the decision of player 2 is closer to the inclination of player 1 than his inclination was. By virtue of (6), we have:

$$
\begin{gathered}
\int_{I_{1 \rightarrow 2}}\left|i_{2}-i_{1}\right| d \lambda^{2}=\frac{1}{3} . \\
I_{1 \rightarrow 2}^{*}(B)=\left\{\mathbf{i} \in I_{1 \rightarrow 2}:\left|\frac{i_{1}+i_{2}}{2}-i_{1}\right|<\left|i_{2}-i_{1}\right|\right\}=I_{1 \rightarrow 2} .
\end{gathered}
$$

If we calculate the positive influence index $D(B, 1 \rightarrow 2)$ of player 1 on player 2 under the influence function $B$, then from (5) and (7) we have

$$
\int_{I_{1 \rightarrow 2}^{*}(B)}\left[\left|i_{2}-i_{1}\right|-\left|(B(\mathbf{i}))_{2}-i_{1}\right|\right] d \lambda^{2}=\frac{1}{2} \cdot \int_{I_{1 \rightarrow 2}}\left|i_{2}-i_{1}\right| d \lambda^{2}
$$

and therefore

$$
D(B, 1 \rightarrow 2)=\frac{1}{2}
$$

Let us assume now that the decision of player 2 is as extreme with respect to the inclination of player 1 as possible, that is,

$$
\left(B^{\prime}\left(i_{1}, i_{2}\right)\right)_{1}=i_{1}, \quad\left(B^{\prime}\left(i_{1}, i_{2}\right)\right)_{2}=\left\{\begin{array}{ll}
0 & \text { if } i_{1} \geq \frac{1}{2} \\
1 & \text { if } 0 \leq i_{1}<\frac{1}{2}
\end{array} \quad \text { for each }\left(i_{1}, i_{2}\right) \in[0,1]^{2} .\right.
$$

In this case, $I_{1 \rightarrow 2}^{*}\left(B^{\prime}\right)=\emptyset$, and therefore $D\left(B^{\prime}, 1 \rightarrow 2\right)=0$.

Another influence function which we study is defined as follows:

$$
\left(B^{\prime \prime}\left(i_{1}, i_{2}\right)\right)_{1}=i_{1}, \quad\left(B^{\prime \prime}\left(i_{1}, i_{2}\right)\right)_{2}=\left\{\begin{array}{ll}
i_{2} & \text { if }\left|i_{2}-i_{1}\right| \leq \frac{1}{2} \\
i_{1}+\frac{1}{2} & \text { if } i_{2}>i_{1}+\frac{1}{2} \\
i_{1}-\frac{1}{2} & \text { if } i_{2}<i_{1}-\frac{1}{2}
\end{array} \quad \text { for each }\left(i_{1}, i_{2}\right) \in[0,1]^{2} .\right.
$$


According to (9), if the difference between the inclinations of players 1 and 2 is not greater than $\frac{1}{2}$, then player 2 decides according to his own inclination, otherwise the decision of player 2 is of an exact distance $\frac{1}{2}$ from the inclination of player 1 . It is a kind of a partial influence meaning that player 2 likes to decide according to his own inclination if his inclination is not that far from the inclination of player 1 , and he 'adjusts' his inclination if there is a rather serious difference between the inclinations of both players. We have

$$
\begin{aligned}
I_{1 \rightarrow 2}^{*}\left(B^{\prime \prime}\right) & =\left\{\left(i_{1}, i_{2}\right) \in I_{1 \rightarrow 2}:\left|\left(B^{\prime \prime}(\mathbf{i})\right)_{2}-i_{1}\right|<\left|i_{2}-i_{1}\right|\right\} \\
& =\left\{\left(i_{1}, i_{2}\right) \in[0,1]^{2}: i_{2}>i_{1}+\frac{1}{2}\right\} \cup\left\{\left(i_{1}, i_{2}\right) \in[0,1]^{2}: i_{2}<i_{1}-\frac{1}{2}\right\} .
\end{aligned}
$$

Figure 2 presents the set $I_{1 \rightarrow 2}^{*}\left(B^{\prime \prime}\right)$ which consists of all the points $\left(i_{1}, i_{2}\right)$ of the two triangles without the lines $i_{2}=i_{1}+\frac{1}{2}$ and $i_{2}=i_{1}-\frac{1}{2}$.

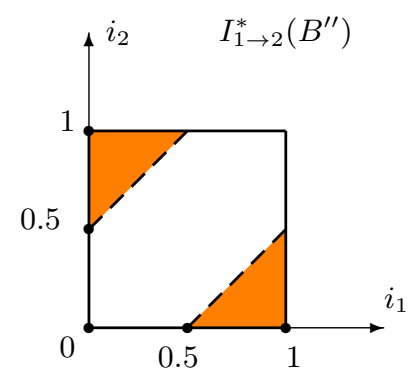

Fig. 2. The set $I_{1 \rightarrow 2}^{*}\left(B^{\prime \prime}\right)$ in the 2-player example

$$
\begin{gathered}
\int_{I_{1 \rightarrow 2}^{*}\left(B^{\prime \prime}\right)}\left[\left|i_{2}-i_{1}\right|-\left|\left(B^{\prime \prime}(\mathbf{i})\right)_{2}-i_{1}\right|\right] d \lambda^{2}= \\
=\int_{0}^{\frac{1}{2}} \int_{i_{1}+\frac{1}{2}}^{1}\left(i_{2}-i_{1}-\frac{1}{2}\right) d \lambda_{2} d \lambda_{1}+\int_{\frac{1}{2}}^{1} \int_{0}^{i_{1}-\frac{1}{2}}\left(i_{1}-i_{2}-\frac{1}{2}\right) d \lambda_{2} d \lambda_{1}=\frac{1}{24} .
\end{gathered}
$$

Hence, the positive influence index of player 1 on player 2 under $B^{\prime \prime}$ is

$$
D\left(B^{\prime \prime}, 1 \rightarrow 2\right)=\frac{1}{8}
$$

Finally, we analyze the following family of influence functions:

$$
B^{x}\left(i_{1}, i_{2}\right)=\left(i_{1}, x\right) \text { for each }\left(i_{1}, i_{2}\right) \in[0,1]^{2},
$$

where $x \in[0,1]$. This means that the first player always decides according to his own inclination, and the second player always chooses the action $x$. There can be several reasons for choosing always the same action $x$. For instance, a certain fixed percent of funding is coherent with a policy of a given foundation, or the experience shows that a certain percent usually represents well real needs to cover expenses of an average project within a given framework, etc. In this case, we cannot really speak of the influence of 
player 1 on player 2, but rather of a kind of a 'luck' rate of player 1, meaning that player 2 makes a decision which is closer to the inclination of player 1 than his inclination was. Nevertheless, we like to calculate the influence index for this example, because such a 'constant decision' is not that rare in real life situations. Moreover, we want to show which decision of a referee makes the other referee 'most lucky'. In this example, we have

$$
I_{1 \rightarrow 2}^{*}\left(B^{x}\right)=\left\{\mathbf{i} \in I_{1 \rightarrow 2}:\left|x-i_{1}\right|<\left|i_{2}-i_{1}\right|\right\}
$$

which is presented in Figure 3.

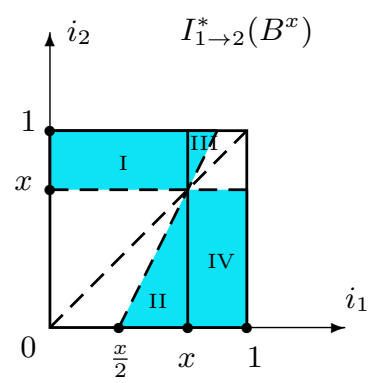

Fig. 3. The set $I_{1 \rightarrow 2}^{*}\left(B^{x}\right)$ in the 2-player example

This set $I_{1 \rightarrow 2}^{*}\left(B^{x}\right)$ consists of the following four subsets:

$$
\begin{gathered}
I_{1 \rightarrow 2}^{I}\left(B^{x}\right)=\left\{\left(i_{1}, i_{2}\right) \in[0,1]^{2}:\left[0 \leq i_{1} \leq x \wedge x<i_{2} \geq 1\right]\right\} \\
I_{1 \rightarrow 2}^{I I}\left(B^{x}\right)=\left\{\left(i_{1}, i_{2}\right) \in[0,1]^{2}:\left[\frac{x}{2}<i_{1} \leq x \wedge 0 \leq i_{2}<2 i_{1}-x\right]\right\} \\
I_{1 \rightarrow 2}^{I I I}\left(B^{x}\right)=\left\{\left(i_{1}, i_{2}\right) \in[0,1]^{2}:\left[x<i_{1}<\frac{1+x}{2} \wedge 2 i_{1}-x<i_{2} \leq 1\right]\right\} \\
I_{1 \rightarrow 2}^{I V}\left(B^{x}\right)=\left\{\left(i_{1}, i_{2}\right) \in[0,1]^{2}:\left[x<i_{1} \leq 1 \wedge 0 \leq i_{2}<x\right]\right\} .
\end{gathered}
$$

The positive influence index $D\left(B^{x}, 1 \rightarrow 2\right)$ of player 1 on player 2 under $B^{x}$ is

$$
\begin{aligned}
D\left(B^{x}, 1 \rightarrow 2\right) & =\frac{\int_{I_{1 \rightarrow 2}^{*}\left(B^{x}\right)}\left[\left|i_{2}-i_{1}\right|-\left|\left(B^{x}(\mathbf{i})\right)_{2}-i_{1}\right|\right] d \lambda^{2}}{\int_{I_{1 \rightarrow 2}}\left|i_{2}-i_{1}\right| d \lambda^{2}} \\
& =3 \sum_{L \in\{I, I I, I I I, I V\}} \int_{I_{1 \rightarrow 2}^{L}\left(B^{x}\right)}\left[\left|i_{2}-i_{1}\right|-\left|x-i_{1}\right|\right] d \lambda^{2}
\end{aligned}
$$

After calculating the four integrals, we get

$$
\begin{aligned}
D\left(B^{x}, 1 \rightarrow 2\right) & =3\left[\frac{1}{2} x(x-1)^{2}+\frac{1}{12} x^{3}+\frac{1}{12}(1-x)^{3}+\frac{1}{2} x^{2}(1-x)\right] \\
& =\frac{1}{4}\left(1+3 x-3 x^{2}\right) .
\end{aligned}
$$

Note that

$$
\frac{1}{4} \leq D\left(B^{x}, 1 \rightarrow 2\right) \leq \frac{7}{16} \text { for all } x \in[0,1]
$$




$$
D\left(B^{0}, 1 \rightarrow 2\right)=D\left(B^{1}, 1 \rightarrow 2\right)=\frac{1}{4}
$$

and

$$
\arg \max _{x \in[0,1]} D\left(B^{x}, 1 \rightarrow 2\right)=\frac{1}{2}
$$

which means that the positive influence index of player 1 on player 2 is minimal if player 2 makes the 'extreme' decisions, and it is maximal if player 2 proposes to assign $50 \%$ of the requested budget to the evaluated project, no matter what the inclinations of both referees are.

\subsection{The three-player example}

Let us consider a three-player example which is similar to the 2-player example presented in Section 3.1, except that the committee evaluating a scientific project consists now of 3 referees, that is, $N=\{1,2,3\}$. Suppose $S=\{1,3\}, j=2$, and we measure the influence of coalition $S$ on player $j$. We omit braces for sets, i.e., in particular, $\{1,3\}$ will be written 13. We have $I=[0,1]^{3}$, that is, $\mathbf{i}=\left(i_{1}, i_{2}, i_{3}\right), \mathbf{i}^{[S]}=\left(i_{[13]}, i_{2}\right)$. Moreover,

$$
\begin{gathered}
I_{13}=\left\{\left(i_{1}, i_{2}, i_{3}\right) \in[0,1]^{3}: i_{1}=i_{3}\right\} \\
I_{13 \rightarrow 2}^{[13]}=\left\{\left(i_{13}, i_{2}\right) \in[0,1]^{2}: i_{2} \neq i_{13}\right\}
\end{gathered}
$$

Figure 4 shows the respective sets of influence vectors: $I, I_{13}$, and $I_{13 \rightarrow 2}^{[13]}$.

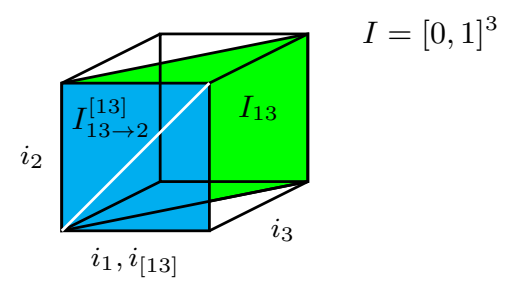

Fig. 4. The sets $I, I_{13}$, and $I_{13 \rightarrow 2}^{[13]}$ in the 3-player example

We define the influence function $\widetilde{B}:[0,1]^{3} \rightarrow[0,1]^{3}$ as follows:

$$
\widetilde{B}\left(i_{1}, i_{2}, i_{3}\right)=\left(i_{1}, \frac{i_{1}+2 i_{2}+i_{3}}{4}, i_{2}\right) \text { for each }\left(i_{1}, i_{2}, i_{3}\right) \in[0,1]^{3} .
$$

Note that the influence function $B:[0,1]^{2} \rightarrow[0,1]^{2}$ defined by (7) in Section 3.1 is the corresponding influence function for $\widetilde{B}$, i.e.,

$$
B\left(i_{13}, i_{2}\right)=\left(i_{13}, \frac{i_{13}+i_{2}}{2}\right) \text { for each }\left(i_{13}, i_{2}\right) \in[0,1]^{2}
$$

Moreover,

$$
I_{13 \rightarrow 2}^{*[13]}(\widetilde{B})=\left\{\left(i_{13}, i_{2}\right) \in I_{13 \rightarrow 2}^{[13]}:\left|\frac{i_{13}+i_{2}}{2}-i_{13}\right|<\left|i_{2}-i_{13}\right|\right\}=I_{13 \rightarrow 2}^{[13]} .
$$


We get therefore

$$
D(\widetilde{B}, 13 \rightarrow 2)=D(B,[13] \rightarrow 2)=\frac{1}{2} .
$$

It is easy to show that such a relation holds for a general case, i.e., there is the equivalence between the influence index of a coalition on a player in a social network and the analogous influence index in a corresponding network in which the coalition in question is treated as one player.

\section{The unanimous influence functions}

When we describe phenomena of a positive influence in terms of a corresponding influence function, it is usually sufficient to restrict the analysis to unanimous functions.

Definition 2 The influence function $B \in \mathcal{B}$ is unanimous if $B(x, x, \ldots, x)=(x, x, \ldots, x)$ for each $x \in[a, b]$, and unanimous on the boundaries if this holds only for $x=a$ and $x=b$.

Obviously, influence functions depicting a negative (opposite) influence should not satisfy the unanimous properties. In Section 3.1, the influence functions $B$ and $B^{\prime \prime}$ defined in (7) and (9), respectively, are unanimous, as well as the function $\widetilde{B}$ presented in Section 3.2 (see (11)). We present several other examples of influence functions that are unanimous. These influence functions have been introduced in Grabisch and Rusinowska (2010a, 2010b, [30,31]) and are now generalized for the influence model with a continuum of actions.

The first influence function we like to mention is the majority influence function. If the players decide according to this function, then in case a majority of players has an inclination $x$, all players decide for $x$, and if not, then each player decides according to his own inclination.

Definition 3 (The majority function) Let $n \geq t>\left\lfloor\frac{n}{2}\right\rfloor$, and introduce for any $\mathbf{i} \in I$ and $x \in[a, b]$, the set $i^{x}:=\left\{k \in N: i_{k}=x\right\}$. The majority influence function Maj $^{[t]} \in \mathcal{B}$ is defined by

$$
\left(\operatorname{Maj}^{[t]}(\mathbf{i})\right)_{j}:=\left\{\begin{array}{ll}
x, & \text { if } \exists x \in[a, b]\left[\left|i^{x}\right| \geq t\right] \\
i_{j}, & \text { otherwise }
\end{array}, \quad \forall \mathbf{i} \in I, \quad \forall j \in N .\right.
$$

Another influence function analyzed in this paper is the guru function which simply means that when a guru exists, every player always follows the guru.

Definition 4 (The guru function) Let $\widetilde{k} \in N$ be a particular player called the guru. The guru influence function Gur ${ }^{[\widetilde{k}]} \in \mathcal{B}$ is defined by

$$
\left(\operatorname{Gur}^{[\widetilde{k}]}(\mathbf{i})\right)_{j}=i_{\widetilde{k}}, \quad \forall \mathbf{i} \in I, \quad \forall j \in N .
$$

Also the identity function, according to which every player always follows himself, is a particular influence function where no influence is visible.

Definition 5 (The identity function) The identity function $\mathrm{Id} \in \mathcal{B}$ is defined by

$$
\operatorname{Id}(\mathbf{i})=\mathbf{i}, \quad \forall \mathbf{i} \in I .
$$


Finally, we like to mention the mass psychology function. According to this influence function, if there is a sufficiently high number of players with inclination $x$, none of them will decide differently than $x$, and they will possibly attract other players to choose $x$.

Definition 6 (The mass psychology function) Let $t \in(0, n]$ and $x \in[a, b]$. Functions $B \in \mathcal{B}$ satisfying for each $\mathbf{i} \in I$

$$
\text { if }\left|i^{x}\right| \geq t, \text { then }(B(\mathbf{i}))^{x} \supseteq i^{x}
$$

are called mass psychology influence functions. We denote by $\mathcal{B}^{[x, t]}$ the set of such influence functions.

It is straightforward to calculate the influence indices for some particular cases. We omit the proofs, since they are similar to those in the discrete case; see Grabisch and Rusinowska (2010b, [31]).

Proposition 1 (i) Let $n \geq t>\left\lfloor\frac{n}{2}\right\rfloor$. For each $\emptyset \neq S \subset N$ such that $s \geq t$, and for each $j \in N \backslash S, D\left(\right.$ Maj $\left.^{[t]}, S \rightarrow j\right)=1$.

If $t=n$, then for each $\emptyset \neq S \subset N$ and $j \in N \backslash S, D\left(\mathrm{Maj}^{[t]}, S \rightarrow j\right)=0$.

(ii) Let $\widetilde{k} \in N$. For each $\emptyset \neq S \subseteq N$ such that $\widetilde{k} \in S$, and for each $j \in N \backslash S$, $D\left(\operatorname{Gur}^{[\widetilde{k}]}, S \rightarrow j\right)=1$.

(iii) For each $\emptyset \neq S \subseteq N$ and $j \in N \backslash S, D(\mathrm{Id}, S \rightarrow j)=0$.

(iv) Let $t \in(0, n]$ and $x \in[a, b]$ be fixed and consider any influence function $B$ in $\mathcal{B}^{[x, t]}$. There exists $B \in \mathcal{B}^{[x, t]}$ such that for each $\emptyset \neq S \subseteq N$ and $j \in N \backslash S, D(B, S \rightarrow j)=0$. For each $\emptyset \neq S \subseteq N$ such that $s>n-t, t>1$, and $j \in N \backslash S$, there exists $B \in \mathcal{B}^{[x, t]}$ such that $D(B, S \rightarrow j)=1$.

\section{Fixed points of an influence function}

A central notion in studying influence functions is the notion of fixed points, that is, those inclination vectors which, despite the influence between players, remain invariant.

Definition 7 An inclination vector $\mathbf{i} \in I$ is a fixed point under $B \in \mathcal{B}$ if $B(\mathbf{i})=\mathbf{i}$. The set of fixed points under $B$ will be denoted by $I^{F P}(B)$.

Fixed points are of fundamental interest in the study of the convergence of the influence process, when several steps of influence are considered. Indeed, we may consider that there are several steps of discussion before every voter decides, and in each step of discussion, when every player reveals his opinion, influence occurs. Assuming that the same mechanism of influence depicted by the influence function $B$ acts in each step, the successive vectors of the opinion of the voters, starting from the original inclination $\mathbf{i}$, are: $\mathbf{i}, B(\mathbf{i}), B(B(\mathbf{i}))=: B^{(2)}(\mathbf{i}), B^{(3)}(\mathbf{i})$, etc. Let us adopt the following convenient notation: $\mathbf{i}(0):=\mathbf{i}$ is the (initial) inclination vector, then $\mathbf{i}(1):=B(\mathbf{i})$ is the opinion vector after one step of influence, etc., and in general $\mathbf{i}(k):=B^{(k)}(\mathbf{i})$ is the opinion vector after $k$ steps.

Suppose that $B$ is continuous on $I$, consider some $\mathbf{i} \in I$ and the sequence $\mathbf{i}(0), \mathbf{i}(1), \ldots$, $\mathbf{i}(k), \ldots$ Suppose that the sequence $\mathbf{i}(k)$ converges to some $\mathbf{i}^{*}$. Then by continuity, $B(\mathbf{i}(k))$ converges to $B\left(\mathbf{i}^{*}\right)=\mathbf{i}^{*}$. Therefore, $\mathbf{i}^{*}$ is a fixed point of $B$. Conversely, if $\mathbf{i}$ is a fixed point, then

$$
\mathbf{i}=B(\mathbf{i})=B^{(2)}(\mathbf{i})=\cdots=B^{(\infty)}(\mathbf{i}):=\lim _{k \rightarrow \infty} B^{(k)}(\mathbf{i})
$$


Consequently, if the $\operatorname{limit}_{k \rightarrow \infty} B^{(k)}(\mathbf{i})$ exists for every $\mathbf{i} \in I$, then

$$
B^{(\infty)}(I)=I^{F P}(B) \text {. }
$$

Therefore, knowing the set of fixed points of $B$ is of primary importance.

In this section, we give general results on this issue, borrowing the main results of fixed point theory (see, e.g., Granas and Dugundji (2003) [32]). Moreover, we determine the set of fixed points for some of the examples given in this paper. A deeper study can be done only for specific families of influence functions, but this is a topic of further research.

A first remark is that if $B$ is unanimous for some $x \in[a, b]$, that is, $B(x, x, \ldots, x)=$ $(x, x, \ldots, x)$, then $(x, x, \ldots, x)$ is a fixed point. Hence, for a unanimous function $B, I^{F P}(B)$ contains the diagonal of $[a, b]^{n}$. In fact, by the Brouwer theorem, if $B$ is a continuous function, then the set $I^{F P}(B)$ is nonempty and compact.

Existence and uniqueness of fixed points can be proved using the Banach theorem: if the function $B$ is contracting, that is, if $d\left(B(\mathbf{i}), B\left(\mathbf{i}^{\prime}\right)\right)<d\left(\mathbf{i}, \mathbf{i}^{\prime}\right)$ holds for any distinct vectors $\mathbf{i}, \mathbf{i}^{\prime} \in I$ and a given metric distance $d$, then there exists a unique fixed point. However, remark that influence functions being unanimous for at least two vectors $\mathbf{i}, \mathbf{i}^{\prime}$ cannot be contracting, and since unanimity is a rather natural property for influence functions (at least for a positive influence), this result is of little interest.

We have the following simple result. Let $I_{S \rightarrow j}$ denote the set of all $n$-inclination vectors of potential positive influence of $S$ on $j$, i.e.,

$$
I_{S \rightarrow j}:=\left\{\mathbf{i} \in I_{S}: i_{j} \neq i_{S}\right\}
$$

and let $I_{S \rightarrow j}^{*}(B)$ be the set of all $n$-inclination vectors of positive influence of $S$ on $j$ under $B$, i.e.,

$$
I_{S \rightarrow j}^{*}(B):=\left\{\mathbf{i} \in I_{S}:\left[i_{j} \neq i_{S} \wedge\left|(B(\mathbf{i}))_{j}-i_{S}\right|<\left|i_{j}-i_{S}\right|\right]\right\} .
$$

Proposition 2 For any influence function $B$,

$$
I^{F P}(B) \subseteq I \backslash \bigcup_{S, j} I_{S \rightarrow j}^{*}(B)
$$

Proof: Suppose that the inclusion does not hold, i.e., there is $\mathbf{i} \in I$ such that $\mathbf{i} \in I^{F P}(B)$ and $\mathbf{i} \in \bigcup_{S, j} I_{S \rightarrow j}^{*}(B)$. Hence, $B(\mathbf{i})=\mathbf{i}$ and there is $S$ and $j$ such that $\mathbf{i} \in I_{S \rightarrow j}^{*}(B)$. This means that $i_{j} \neq i_{S}$ and $\left|(B(\mathbf{i}))_{j}-i_{S}\right|<\left|i_{j}-i_{S}\right|$, while $(B(\mathbf{i}))_{j}=i_{j}$, a contradiction.

Note that equality does not hold in general (see Example 2 below, function $B^{\prime}$ ), but it may hold for some cases (see Examples 1 and 2, function $B^{\prime \prime}$ ).

We end this section by investigating the set of fixed points for several examples of influence functions introduced above.

Example 1 For the influence function $B$ defined by (7) in Section 3.1, the set of fixed points is

$$
I^{F P}(B)=\left\{\left(i_{1}, i_{2}\right) \in[0,1]^{2}: i_{1}=i_{2}\right\} .
$$

Note that, for this example

$$
I^{F P}(B)=I \backslash\left(I_{1 \rightarrow 2}^{*}(B) \cup I_{2 \rightarrow 1}^{*}(B)\right) .
$$


For the function $\widetilde{B}$ defined by (11) in the three-player example presented in Section 3.2, we have

$$
I^{F P}(\widetilde{B})=\left\{\left(i_{1}, i_{2}, i_{3}\right) \in[0,1]^{3}: i_{1}=i_{2}=i_{3}\right\}
$$

Example 2 Let us calculate the sets of fixed points for the remaining functions of Section 3.1, i.e., the functions $B^{\prime}, B^{\prime \prime}$, and $B^{x}$ defined by (8), (9), and (10), respectively. We have

$$
\begin{gathered}
I^{F P}\left(B^{\prime}\right)=\left\{\left(i_{1}, 0\right): i_{1} \geq \frac{1}{2}\right\} \cup\left\{\left(i_{1}, 1\right): 0 \leq i_{1}<\frac{1}{2}\right\} \\
I^{F P}\left(B^{\prime \prime}\right)=\left\{\left(i_{1}, i_{2}\right) \in[0,1]^{2}: i_{1}-\frac{1}{2} \leq i_{2} \leq i_{1}+\frac{1}{2}\right\} \\
I^{F P}\left(B^{x}\right)=\left\{\left(i_{1}, x\right): 0 \leq i_{1} \leq 1\right\} .
\end{gathered}
$$

Note that

$$
I^{F P}\left(B^{\prime \prime}\right)=I \backslash\left(I_{1 \rightarrow 2}^{*}\left(B^{\prime \prime}\right) \cup I_{2 \rightarrow 1}^{*}\left(B^{\prime \prime}\right)\right) .
$$

However, we have $I_{1 \rightarrow 2}^{*}\left(B^{\prime}\right)=I_{2 \rightarrow 1}^{*}\left(B^{\prime}\right)=\emptyset$, but $I^{F P}\left(B^{\prime}\right) \neq I$.

Example 3 For the unanimous functions presented in Section 4, the sets of fixed points are equal to

$$
\begin{gathered}
I^{F P}(\mathrm{Id})=I, \quad I^{F P}\left(\operatorname{Gur}^{[\widetilde{k}]}\right)=\left\{\mathbf{i} \in I: \forall j, k \in N\left[i_{j}=i_{k}\right]\right\} \\
I^{F P}\left(\mathrm{Maj}^{[t]}\right)=\left\{\mathbf{i} \in I: \forall j, k \in N\left[i_{j}=i_{k}\right]\right\} \cup\left\{\mathbf{i} \in I: \forall x \in[a, b]\left[\left|i^{x}\right|<t\right]\right\} .
\end{gathered}
$$

\section{$6 \quad$ Linear influence functions}

An important particular case is when an influence function $B$ is linear, that is, it can be written as a matrix $\mathbf{B}=\left[b_{i j}\right]_{i, j \in N}$, and then we write $\mathbf{B i}$ instead of $B(\mathbf{i})$. We assume throughout this section that the inclination vectors and decision vectors are column vectors and that $I=[0,1]^{n}$.

\subsection{Examples and general facts}

Example 4 The influence function $B$ defined by (7) in Section 3.1 is linear. For this function, the corresponding matrix is equal to

$$
\mathbf{B}=\left[\begin{array}{ll}
1 & 0 \\
\frac{1}{2} & \frac{1}{2}
\end{array}\right]
$$

Also the function $\widetilde{B}$ defined by (11) in the three-player example presented in Section 3.2 is linear, and

$$
\widetilde{\mathbf{B}}=\left[\begin{array}{lll}
1 & 0 & 0 \\
\frac{1}{4} & \frac{1}{2} & \frac{1}{4} \\
0 & 1 & 0
\end{array}\right]
$$


The guru influence function Gur ${ }^{[\widetilde{k}]}$ is also linear, with matrix

$$
\operatorname{Gur}^{[\widetilde{k}]}=\left[\begin{array}{ccccc}
0 & \cdots & 1 & \cdots & 0 \\
\cdots & \cdots & \vdots & \cdots & \cdots \\
0 & \cdots & 1 & \cdots & 0
\end{array}\right]
$$

i.e., $\operatorname{Gur}^{[\widetilde{k}]}$ has the form $b_{j \widetilde{k}}=1$ and $b_{j k}=0$ for $k \neq \widetilde{k}, j \in N$. Obviously, the identity function Id corresponds to the identity matrix.

Let us denote by $\mathbf{1 , 0}$ the $n$-dimensional vectors having all components equal to 1 (respectively, 0). A first remark is that any linear influence function is unanimous on the value 0 , since $\mathbf{B} \mathbf{0}=\mathbf{0}$. Saying that $\mathbf{B}$ is unanimous on the value 1 means that $\mathbf{B} \mathbf{1}=\mathbf{1}$, which implies $\sum_{k=1}^{n} b_{j k}=1$ for each row $\mathbf{b}_{j}$. Another noteworthy fact is that the entries of any influence matrix $\mathbf{B}$ should be nonnegative, for, if it would exist some negative entry, the resulting decision vector $\mathbf{B i}$ might have negative components, which is impossible since $I=[0,1]^{n}$. These observations are summarized in the next lemma.

Lemma 2 Let $\mathbf{B}=\left[b_{i j}\right]_{i, j=1, \ldots, n}$ be the matrix of some influence function.

(i) $b_{i j} \geq 0$ for $i, j=1, \ldots, n$.

(ii) $\mathbf{B}$ is unanimous on the value 0 .

(iii) $\mathbf{B}$ is unanimous on the value 1 if and only if $\mathbf{B}$ is unanimous if and only if $\sum_{k=1}^{n} b_{j k}=1$ for each row $\mathbf{b}_{j}$.

An important consequence is that the set of unanimous linear influence functions on $[0,1]$ corresponds to the set of (row) stochastic matrices. It is well known that the set of stochastic matrices is closed under matrix multiplication, and if $\mathbf{B}$ is a stochastic matrix then its inverse, whenever it exists, is also a stochastic matrix (and therefore a linear unanimous influence function).

A remarkable feature of linear functions is that they map polyhedra to polyhedra. Since $I$ is a polyhedron, it follows that all $\mathbf{B}^{k}(I), k=1,2, \ldots$ are polyhedra. Moreover, computing $\mathbf{B}^{k}(I)$ amounts to computing only $\mathbf{B}^{k}(\mathbf{i})$ for all vertices of $I$ since we have the property

$$
\mathbf{B}^{k}(I)=\operatorname{conv}\left(\mathbf{B}^{k}(\operatorname{ext}(I))\right)
$$

where $\operatorname{ext}(\cdot)$ and $\operatorname{conv}(\cdot)$ denote the vertices of a polyhedron and the convex closure of a set.

\subsection{Convergence and fixed points}

By definition, $\mathbf{i}$ is a fixed point of $\mathbf{B}$ if and only if $\mathbf{i}$ is a solution of the linear system $(\mathbf{B}-$ $\mathbf{I}) \mathbf{i}=\mathbf{0}$, where $\mathbf{I}$ is the identity matrix (equivalently, if $\mathbf{i}$ is an eigenvector associated with the eigenvalue 1). If $\mathbf{B}$ is a stochastic matrix, the problem of finding fixed points is wellknown, and is related to Markov chain theory; see DeGroot (1974, [18]), Jackson (2008, [37], Chapter 8.3), Golub and Jackson (2010, [29]). We give here a basic explanation and refer to Jackson for details. As before, we use the notation $\mathbf{i}(0):=\mathbf{i}, \mathbf{i}(1)=\mathbf{B i}, \ldots, \mathbf{i}(k)=$ $\mathbf{B}^{k} \mathbf{i}$, etc.

First, we introduce some special properties of stochastic matrices. To each matrix B we associate a digraph $\Gamma$ whose nodes are players, and a directed edge exists from $j$ to $k$ 
if and only if $b_{j k}>0$ (then we say that $k$ is a successor of $j$ ). A path in $\Gamma$ is a sequence of nodes $j_{1}, \ldots, j_{l}$ such that $j_{k+1}$ is a successor of $j_{k}$ for $k=1, \ldots, l-1$. A cycle is a path, where the first and the last nodes coincide, and its length is the number of its edges. A (strongly) connected component is any subset $S$ of nodes such that there is a path from any node to any other node of $S$, and which is maximal for this property. If the matrix has only one connected component (which is then $N$ ), then it is said to be irreducible. A connected component is closed if there is no edge going outside it. A closed connected component is periodic of period $p$ if $p$ is the greatest common divisor of the length of all cycles in it. If $p=1$, then the connected component is said to be aperiodic (if the matrix is irreducible, then we say that the matrix itself is aperiodic).

Let us now turn to the study of convergence. We say that a consensus is reached for a given $\mathbf{i}$ if $\lim _{k \rightarrow \infty} \mathbf{i}(k)=\left[i^{*} i^{*} \cdots i^{*}\right]^{T}=: \mathbf{i}^{*}$ for some $i^{*} \in[0,1]$. It is easy to see that reaching consensus for every $\mathbf{i}$ is equivalent to the fact that $\mathbf{B}^{\infty}:=\lim _{k \rightarrow \infty} \mathbf{B}^{k}$ exists and is equal to

$$
\mathrm{B}^{\infty}=\left[\begin{array}{c}
\pi \\
\pi \\
\vdots \\
\pi
\end{array}\right]
$$

where $\boldsymbol{\pi}=\left[\pi_{1} \ldots \pi_{n}\right]$ is a probabilistic row vector. In this case, $\mathbf{i}^{*}=\mathbf{B}^{\infty} \mathbf{i}(0)$. Now, $\boldsymbol{\pi}$ is obtained as the solution of the eigenvalue equation $\boldsymbol{\pi} \mathbf{B}=\boldsymbol{\pi}$ provided $\mathbf{B}$ has only one aperiodic closed connected component (in particular, if $\mathbf{B}$ is irreducible and aperiodic). Indeed, if $\mathbf{x}$ is a probabilistic row vector, we have $\mathbf{x B}^{\infty}=\pi$, hence $\underbrace{\mathrm{xB}^{\infty} \mathbf{B}}_{\boldsymbol{\pi}}=\pi \mathbf{B}$. The numbers $\pi_{1}, \ldots, \pi_{n}$ can be interpreted as the weight of each agent in the consensus.

The above explanation establishes that a consensus is reached if and only if the matrix has only one closed connected component which is aperiodic (this is Corollary 8.1 in Jackson (2008, [37])). Agents outside the closed connected component converge to the consensus, but they have no weight, i.e., the consensus is independent of their initial opinions. If there are several closed connected components which are aperiodic, then each of them leads to a consensus among the players in the component, with their own weights. The remaining agents converge to a mixture of the different consensus, and have no influence on them (see Theorem 8.3 in Jackson (2008, [37])). If a connected component is periodic, there is no convergence and $\mathbf{i}(k)$ endlessly cycles between $p$ vectors. In summary, in case of convergence, the limit matrix $\mathbf{B}^{\infty}$ has the following structure, assuming players outside connected components are listed first:

$$
\mathbf{B}^{\infty}=\left[\begin{array}{c|ccc}
\mathbf{0} & & \mathbf{P} & \\
\hline & \mathbf{B}_{1}^{\infty} & & \\
\mathbf{0} & & \ddots & \\
& & & \mathbf{B}_{k}^{\infty}
\end{array}\right]
$$

where $\mathbf{B}_{1}^{\infty}, \ldots, \mathbf{B}_{k}^{\infty}$ are row-stochastic matrices with identical rows $\boldsymbol{\pi}_{1}, \ldots, \boldsymbol{\pi}_{k}$ respectively, corresponding to the $k$ closed aperiodic connected components, and the structure of $\mathbf{P}$ depends on the structure of the digraph $\Gamma$.

Let us come back to fixed points. If all closed connected components of $\mathbf{B}$ are aperiodic, then we are in the conditions of applying (16). The matrix $\mathbf{B}^{\infty}$ is given in (21), therefore 
the set of fixed points is

$$
\begin{gathered}
I^{F P}(\mathbf{B})= \\
\left\{\mathbf{i} \in I \mid \mathbf{i}=[\underbrace{\sum_{i=1}^{k} \alpha_{i}^{1} \overline{\boldsymbol{\pi}}_{i} \mathbf{i}_{0}, \ldots, \sum_{i=1}^{k} \alpha_{i}^{l} \overline{\boldsymbol{\pi}}_{i} \mathbf{i}_{0}}_{\text {remaining agents }}, \underbrace{\overline{\boldsymbol{\pi}}_{1} \mathbf{i}_{0}, \ldots, \overline{\boldsymbol{\pi}}_{1} \mathbf{i}_{0}}_{\text {agents in } \mathbf{B}_{1}}, \underbrace{\left.\left.\overline{\boldsymbol{\pi}}_{2} \mathbf{i}_{0}, \ldots, \overline{\boldsymbol{\pi}}_{2} \mathbf{i}_{0}, \ldots, \ldots, \overline{\boldsymbol{\pi}}_{k} \mathbf{i}_{0}\right], \mathbf{i}_{0} \in I\right\}}_{\text {agents in } \mathbf{B}_{2}}\right.
\end{gathered}
$$

where $\overline{\boldsymbol{\pi}}_{i}=\left[\ldots 0 \ldots \boldsymbol{\pi}_{i} \ldots 0 \ldots\right]$, the zeros corresponding to players outside connected component $i$. Note that if there is a single closed connected component, then the set of fixed points is the set of consensus vectors, i.e., the diagonal of $I$.

\subsection{Relation with degrees of influence}

Proposition 3 Let $\mathbf{B}$ be unanimous on the boundary 1. Take $S \subseteq N, j \notin S$. S has a positive influence on $j$ for every $\mathbf{i} \in I_{S \rightarrow j}$ if and only if $b_{j j}<1$ and $b_{j k}=0$ for all $k \in N \backslash(S \cup j)$. Moreover, in this case

$$
D(\mathbf{B}, S \rightarrow j)=1-b_{j j} .
$$

Proof: Let $\mathbf{B}$ be arbitrary. There is positive influence of $S$ on $j$ for $\mathbf{i} \in I_{S \rightarrow j}$ if

$$
\left|(\mathbf{B i})_{j}-i_{S}\right|<\left|i_{j}-i_{S}\right| .
$$

By Lemma 2 we find

$$
\begin{aligned}
(\mathbf{B i})_{j}-i_{S} & =i_{S} \sum_{k \in S} b_{j k}+\sum_{k \notin S} b_{j k} i_{k}-i_{S} \\
& =i_{S}\left(-\sum_{k \notin S} b_{j k}\right)+\sum_{k \notin S} b_{j k} i_{k} \\
& =b_{j j}\left(i_{j}-i_{S}\right)+\sum_{\substack{k \notin S \\
k \neq j}} b_{j k}\left(i_{k}-i_{S}\right) .
\end{aligned}
$$

We have to find conditions on $\mathbf{B}$ such that for all $\mathbf{i} \in I_{S \rightarrow j}$, (23) holds, i.e.

$$
\left|b_{j j}\left(i_{j}-i_{S}\right)+\sum_{\substack{k \notin S \\ k \neq j}} b_{j k}\left(i_{k}-i_{S}\right)\right|<\left|i_{j}-i_{S}\right| .
$$

We have

$$
\left|b_{j j}\left(i_{j}-i_{S}\right)+\sum_{\substack{k \notin S \\ k \neq j}} b_{j k}\left(i_{k}-i_{S}\right)\right| \leq\left|b_{j j}\left(i_{j}-i_{S}\right)\right|+\left|\sum_{\substack{k \notin S \\ k \neq j}} b_{j k}\left(i_{k}-i_{S}\right)\right| .
$$

Moreover, $\sum_{\substack{k \notin S \\ k \neq j}} b_{j k}\left(i_{k}-i_{S}\right) \leq \sum_{\substack{k \notin S \\ k \neq j}} b_{j k}$ since $-1 \leq i_{k}-i_{S} \leq 1$ for all $k \neq j, k \notin S$, and equality can be attained by choosing $\mathbf{i}$ with $i_{k}-i_{S}=1$. Therefore, for every $\mathbf{i} \in I_{S \rightarrow j}$

$$
0 \leq\left|b_{j j}\left(i_{j}-i_{S}\right)+\sum_{\substack{k \notin S \\ k \neq j}} b_{j k}\left(i_{k}-i_{S}\right)\right| \leq\left|b_{j j}\left(i_{j}-i_{S}\right)\right|+\sum_{\substack{k \notin S \\ k \neq j}} b_{j k}
$$


The right hand expression and $\left|i_{j}-i_{S}\right|$ being continuous functions of $i_{j}$ on $[0,1]$, we have by $(24)$

$$
0 \leq \lim _{i_{j} \rightarrow i_{S}}\left(\left|b_{j j}\left(i_{j}-i_{S}\right)\right|+\sum_{\substack{k \notin S \\ k \neq j}} b_{j k}\right) \leq \lim _{i_{j} \rightarrow i_{S}}\left|i_{j}-i_{S}\right|
$$

which becomes

$$
0 \leq \sum_{\substack{k \notin S \\ k \neq j}} b_{j k} \leq 0
$$

and yields $b_{j k}=0$ for every $k \in N \backslash(S \cup j)$. Substituting into (24) yields

$$
\left|b_{j j}\left(i_{j}-i_{S}\right)\right|<\left|i_{j}-i_{S}\right|
$$

implying $b_{j j}<1$.

Let us take $\mathbf{B}$ satisfying the above conditions. Then

$$
(\mathbf{B i})_{j}-i_{S}=b_{j j} i_{j}+\sum_{k \in S} b_{j k} i_{S}-i_{S}=b_{j j}\left(i_{j}-i_{S}\right)
$$

Note that for such a $\mathbf{B}$ we have $I_{S \rightarrow j}^{*}(B)=I_{S \rightarrow j}$. Therefore

$$
\begin{aligned}
D(\mathbf{B}, S \rightarrow j) & =\frac{\int_{I_{S \rightarrow j}^{*[S]}(B)}\left(\left|i_{j}-i_{S}\right|-\left|(\mathbf{B i})_{j}-i_{S}\right|\right) d \lambda^{n-s+1}}{\int_{I_{S \rightarrow j}^{[S]}}\left|i_{j}-i_{S}\right| d \lambda^{n-s+1}} \\
& =\frac{\int_{I_{S \rightarrow j}^{[S]}}\left|i_{j}-i_{S}\right|\left(1-b_{j j}\right) d \lambda^{n-s+1}}{\int_{I_{S \rightarrow j}^{[S]}}\left|i_{j}-i_{S}\right| d \lambda^{n-s+1}} \\
& =1-b_{j j} .
\end{aligned}
$$

We recall now the definitions of follower and perfect follower of a given coalition under an arbitrary influence function (not necessarily linear). A follower of a given coalition of agents is a player whose decision is never farther from the inclination of the coalition in question than his inclination was. A player who always decides according to the inclination of the coalition in question is called a perfect follower of that coalition.

Definition 8 Let $\emptyset \neq S \subseteq N$ and $B \in \mathcal{B}$. The set of followers of $S$ under $B$ is given by $F_{B}(S):=\left\{j \in N: \forall \mathbf{i} \in I_{S}\left[\left[i_{j} \neq i_{S} \Rightarrow\left|(B(\mathbf{i}))_{j}-i_{S}\right|<\left|i_{j}-i_{S}\right|\right] \wedge\left[i_{j}=i_{S} \Rightarrow(B(\mathbf{i}))_{j}=i_{S}\right]\right]\right\}$ $F_{B}(\emptyset):=\emptyset$, and the set of perfect followers of $S$ under $B$ is defined as

$$
F_{B}^{\text {per }}(S):=\left\{j \in N: \forall \mathbf{i} \in I_{S}\left[(B(\mathbf{i}))_{j}=i_{S}\right]\right\} .
$$

Remark 1 Note that:

(i) The expression $(\mathbf{B i})_{j}-i_{S}=b_{j j}\left(i_{j}-i_{S}\right)$ shows that: 
- If $b_{j j}=0$, then $(\mathbf{B i})_{j}=i_{S}$, i.e., the influence is full and $j$ is a perfect follower of $S$.

- If $b_{j j}>0$, then $\operatorname{sgn}\left((\mathbf{B i})_{j}-i_{S}\right)=\operatorname{sgn}\left(i_{j}-i_{S}\right)$, which means that due to influence, the decision of $j$ is always going closer to the inclination of $S$, i.e., is in between the original inclination $i_{j}$ and the inclination of $S$. Therefore, $j$ is a follower of $S$.

- If $b_{j j}$ were negative, then $\operatorname{sgn}\left((\mathbf{B i})_{j}-i_{S}\right)=-\operatorname{sgn}\left(i_{j}-i_{S}\right)$, which means that the decision of $j$ would go beyond the inclination of $S$ (we may call this a switch), i.e., the original inclination of $S$ would be in between the original inclination of $j$ and his decision. Since $b_{j j}<0$ is not possible, linear unanimous influence functions cannot represent positive influence with a switch.

(ii) The condition that $\mathbf{B}$ has to fulfill for the influence of $S$ on $j$ concerns only row $j$ of the matrix. Therefore, it is very easy to build/customize a matrix $\mathbf{B}$ such that for some players the influencing coalition is specified (see also Corollary 1 below).

We also recall the definition of a purely influential function.

Definition 9 Let $S, T$ be two disjoint nonempty subsets of $N$. The influence function $B \in \mathcal{B}$ is said to be a purely influential function of $S$ upon $T$ if it satisfies for all $\mathbf{i} \in I_{S}$ :

$$
(B(\mathbf{i}))_{j}= \begin{cases}i_{S} & \text { if } j \in T \\ i_{j} & \text { otherwise. }\end{cases}
$$

The set of such functions is denoted by $\mathcal{B}_{S \rightarrow T}$. The canonical purely influential function of $S$ upon $T$ satisfies in addition $B(\mathbf{i})=\mathbf{i}$ for each $\mathbf{i} \in I \backslash I_{S}$. This function is denoted by $B_{S \rightarrow T}$.

The following is an immediate consequence of Proposition 3.

Corollary 1 Let $S, T$ be two disjoint coalitions. A matrix $\mathbf{B}$ of the form

$$
\begin{aligned}
& b_{j j}=1 \text { and } b_{j k}=0 \text { for } k \neq j, \quad j \notin T \cup S \\
& b_{j k}=0 \text { for } k \notin S, \quad j \in T \\
& b_{j k}=0 \text { for } k \notin S, \quad j \in S
\end{aligned}
$$

is a purely influential function of $S$ upon $T$ (see Figure 5: blank area means 0, elements of each row in the shaded area sum up to 1).

Note that the converse is false, because for such functions nothing is specified when $\mathbf{i}$ is outside $I_{S}$ (in particular they need not be linear). With the above matrix, the behavior of the function outside $I_{S}$ is specified. In particular, the canonical purely influential function of $S$ upon $T$ is not of this form, because outside $I_{S}$, it should be the identity matrix.

It is easy to compute $\mathbf{B}^{n}$ when $\mathbf{B}$ is a purely influential function of $S$ upon $T$. First we order the players so that $S=\{1, \ldots, s\}$ and $T=\{s+1, \ldots, s+t\}$. Let us call $\mathbf{B}_{S}$ the square submatrix corresponding to rows and columns from 1 to $s$, and $\mathbf{B}_{S, T}$ the submatrix corresponding to rows from $s+1$ to $s+t$ and columns from 1 to $s$ (see Figure 6, left). We may denote therefore the matrix by $\mathbf{B}=\left(\mathbf{B}_{S}, \mathbf{B}_{S, T}\right)$. Note that matrices $\mathbf{B}_{S}, \mathbf{B}_{S, T}$ are arbitrary, provided the sum of coefficients on each row is equal to 1 . It is not difficult to see that $\mathbf{B}^{n}$ is still a purely influential function of $S$ upon $T$ with the following structure (see Figure 6, right):

$$
\mathbf{B}^{n}=\left(\mathbf{B}_{S}^{n}, \mathbf{B}_{S, T} \mathbf{B}_{S}^{n-1}\right) .
$$




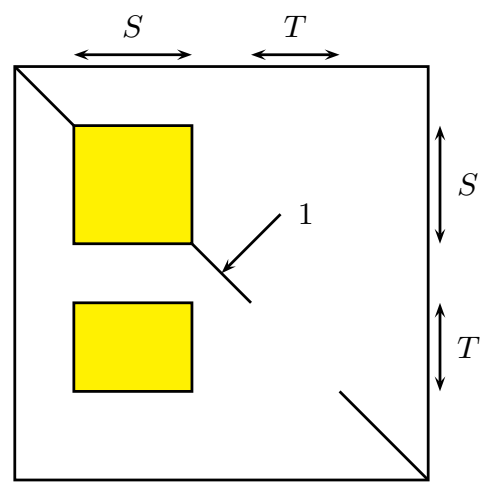

Fig. 5. The matrix B in Corollary 1
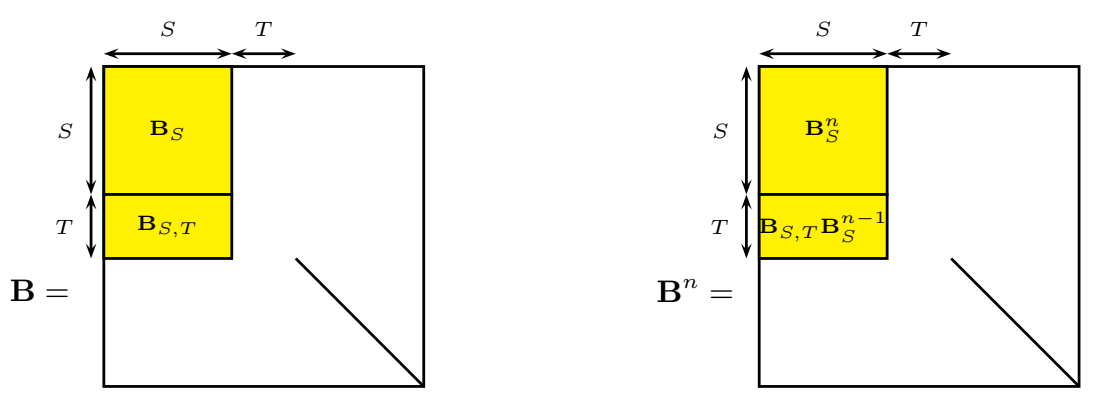

Fig. 6. The structure of matrices $\mathbf{B}$ and $\mathbf{B}^{n}$

\subsection{Related literature}

As mentioned in the Introduction, the linear model of influence is in fact not new. Its first appearance seems to be in DeGroot $(1974,[18])$, who stated the result on convergence for irreducible and aperiodic matrices. Later, several authors investigated more deeply this model, among them, Berger (1981, [4]), DeMarzo et al. (2003, [19]), Jackson (2008, [37]), and Golub and Jackson (2010, [29]).

In a different context, Asavathiratham (2000, [2]) proposed a model which is mathematically equivalent; see also Asavathiratham et al. (2001, [3]). $N$ is a set of agents who must make a yes/no decision. The matrix $\mathbf{B}$ expresses as in our model the weight of each player for influencing another player. However, B multiplies a column vector $\mathbf{x}=\left[\begin{array}{lll}x_{1} & \cdots & x_{n}\end{array}\right]^{T}$ where $x_{i}$ is the probability that player $i$ says 'yes'. Asavathiratham rediscovered DeGroot's results, and shows that, considering sets of 'yes' players as states of a Markov chain, the only terminal states are the consensus states where all players say 'yes' or all players say 'no'. He investigated also in detail the case where $\mathbf{B}$ contains periodic closed connected components.

Lastly, we mention the model proposed by Borm et al. (2002, [11]). In their setting, $N$ is a set of teams, and a digraph $\Gamma$ is used to model competitions between teams: there is an arc from $i$ to $j$ if $i$ defeated $j$. In order to compute a power measure of teams, Borm et al. proposed to consider the matrix $\mathbf{P}$ whose general term is $p_{i j}=\frac{1}{\pi(j)+1}$ if there is an arc from $i$ to $j$ or if $i=j$, and $p_{i j}=0$ otherwise, where $\pi(j)$ is the number of predecessors of $j$ in the digraph. This matrix is column-stochastic, and the power measure $\boldsymbol{\lambda}$ is defined 
as the solution of the eigenvalue equation $\mathbf{P} \boldsymbol{\lambda}=\boldsymbol{\lambda}$. Therefore, $\mathbf{P}^{T}$ can be considered as a particular linear influence matrix, and $\boldsymbol{\lambda}$ exactly corresponds to our vector $\boldsymbol{\pi}$ above, representing the weight of agents in the consensus. The matrix $\mathbf{P}^{T}$ has a very natural interpretation: it says that the opinion of a given player $i$ is the average of opinions of players who are predecessors of him in a given digraph, including himself. We may call the predecessors of an agent his trend-setters.

\section{The negative influence index in the generalized framework}

\subsection{Definition of the index}

The concept of negative influence for the model with a continuum of actions is naturally related to the negative influence defined in the model with a totally ordered set of actions. Under the negative influence in the multi-action framework, the inclination of a player is different from the extreme action(s) of a coalition (that is, the action(s) placed farthest from the inclination of the coalition), and the player's decision comes 'closer' to such an extreme action. We follow here a similar approach.

Let us formalize the concepts of negative influence. For each action $x \in[a, b], M(x)$ is the bound of $[a, b]$ which is farthest from $x$, i.e.:

$$
M(x)= \begin{cases}b, & \text { if } x \in\left[a, \frac{a+b}{2}[\right. \\ a, & \text { if } \left.x \in] \frac{a+b}{2}, b\right] \\ a \text { or } b & \text { if } x=\frac{a+b}{2} .\end{cases}
$$

In the third case, $a$ or $b$ can be chosen, without any influence on the subsequent results. For each $S \subseteq N$ and $j \in N \backslash S$, the set of all $(n-s+1)$-inclination vectors of potential negative influence of $S$ on $j$ is defined as

$$
\widetilde{I}_{S \rightarrow j}^{S S]}:=\left\{\mathbf{i}^{[S]} \in[a, b]^{n-s+1}: i_{j} \neq M\left(i_{S}\right)\right\} .
$$

This means that in any inclination vector of potential negative influence of $S$ on $j$, the inclination of player $j$ is not the extreme action of coalition $S$.

The set of all inclination vectors of negative influence of $S$ on $j$ under given $B$ is defined as

$I_{S \rightarrow j}^{n e g[S]}(B):=\left\{\mathbf{i}^{[S]} \in \widetilde{I}_{S \rightarrow j}^{[S]}:\left[\left|i_{j}-i_{S}\right|<\left|(B(\mathbf{i}))_{j}-i_{S}\right| \wedge\left|i_{j}-M\left(i_{S}\right)\right|>\left|(B(\mathbf{i}))_{j}-M\left(i_{S}\right)\right|\right]\right\}$

$I_{S \rightarrow j}^{n e g[S]}(B)$ is therefore the set of all inclination vectors in $[a, b]^{n-s+1}$ such that the inclination of player $j$ is not the extreme action of coalition $S$, and it satisfies two conditions:

- The first condition of $I_{S \rightarrow j}^{n e g[S]}(B)$ means that the decision of player $j$ goes farther from the inclination of coalition $S$ than his inclination was.

- The second condition says that the decision of player $j$ comes closer to the extreme action of coalition $S$ than his inclination was. Note that in the special case $i_{S}=\frac{a+b}{2}$, this second condition should be modified as follows: $\left|i_{j}-\epsilon\right|>\left|(B(\mathbf{i}))_{j}-\epsilon^{\prime}\right|$, where $\epsilon, \epsilon^{\prime}$ are respectively the bounds closer to $i_{j}$ and closer to $(B(\mathbf{i}))_{j}$. However, this is unimportant since when computing integrals this distinction will disappear. 

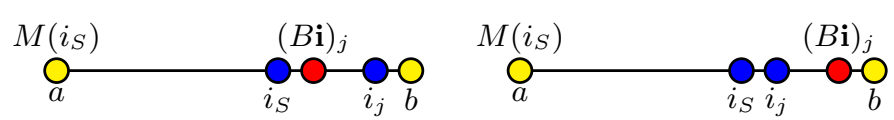

Fig. 7. Violating the conditions for the negative influence

Note that we need both conditions to define the negative influence, since none of these conditions implies the another one. Figure 7 shows the situations in which one of the conditions is not satisfied.

Definition 10 Given $B \in \mathcal{B}$, for each $S \subseteq N, j \in N \backslash S$, the negative influence index of coalition $S$ on player $j$ is defined as

$$
D^{n e g}(B, S \rightarrow j):=\frac{\int_{I_{S \rightarrow j}^{n e g[S]}(B)}\left[\left|i_{j}-M\left(i_{S}\right)\right|-\left|(B(\mathbf{i}))_{j}-M\left(i_{S}\right)\right|\right] d \lambda^{n-s+1}}{\int_{\widetilde{I}_{S \rightarrow j}^{S S}}\left|i_{j}-M\left(i_{S}\right)\right| d \lambda^{n-s+1}} .
$$

The negative influence index defined in (28) is related to the possibility negative influence index for the multi-action model; see Grabisch and Rusinowska (2010b, [31], Definition 2 ). Similarly as for the positive influence, we can calculate the integral in the denominator of definition (28).

Lemma 3 If $I=[a, b]^{n}$, then for each $\emptyset \neq S \subset N$ and $j \in N \backslash S$

$$
\int_{\widetilde{I}_{S \rightarrow j}^{[S]}}\left|i_{j}-M\left(i_{S}\right)\right| d \lambda^{n-s+1}=\frac{(b-a)^{n-s+2}}{2} .
$$

\section{Proof:}

$$
\begin{gathered}
\int_{\widetilde{I}_{S \rightarrow j}^{S S}}\left|i_{j}-M\left(i_{S}\right)\right| d \lambda^{n-s+1}=\int_{a}^{b} \ldots \int_{a}^{b} \int_{a}^{b} \int_{a}^{\frac{a+b}{2}}\left(b-i_{j}\right) d \lambda_{S} d \lambda_{j} d \lambda_{k_{1}} \ldots d \lambda_{k_{n-s-1}}+ \\
+\int_{a}^{b} \ldots \int_{a}^{b} \int_{a}^{b} \int_{\frac{a+b}{2}}^{b}\left(i_{j}-a\right) d \lambda_{S} d \lambda_{j} d \lambda_{k_{1}} \ldots d \lambda_{k_{n-s-1}}=\frac{(b-a)^{n-s+2}}{2}
\end{gathered}
$$

\subsection{Examples of negative influence}

Let us calculate the negative influence indices for some examples for which we have already calculated the positive influence indices.

Example 5 We consider Example 3.1 and the negative influence of player 1 on player 2. The set of potential negative influence is

$$
\widetilde{I}_{1 \rightarrow 2}=\left\{\left(i_{1}, i_{2}\right) \in[0,1]^{2}: i_{2} \neq M\left(i_{1}\right)\right\} .
$$




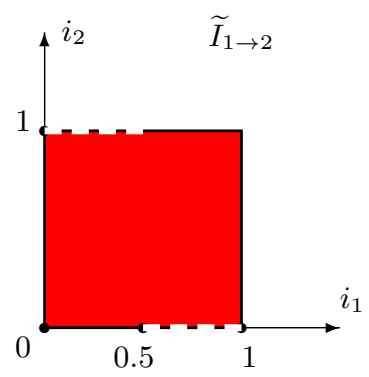

Fig. 8. The set $\widetilde{I}_{1 \rightarrow 2}$ in the 2-player example

The set $\widetilde{I}_{1 \rightarrow 2}$ is presented in Figure 8. It consists of all the points of the square without two intervals: one with $i_{1} \in\left[\frac{1}{2}, 1\right]$ and $i_{2}=0$, and one with $i_{1} \in\left[0, \frac{1}{2}\right]$ and $i_{2}=1$.

By virtue of (29), we have

$$
\int_{\widetilde{I}_{1 \rightarrow 2}}\left|i_{2}-M\left(i_{1}\right)\right| d \lambda^{2}=\frac{1}{2}
$$

For the influence function $B$ defined by $(7)$, we have $I_{1 \rightarrow 2}^{n e g}(B)=\emptyset$, because while there are inclination vectors $\left(i_{1}, i_{2}\right)$ that satisfy the second condition, no inclination vector satisfies the first condition of $I_{1 \rightarrow 2}^{n e g}(B)$. Hence, $D^{\text {neg }}(B, 1 \rightarrow 2)=0$.

Similarly for the function $B^{\prime \prime}$ defined by $(9), I_{1 \rightarrow 2}^{\text {neg }}\left(B^{\prime \prime}\right)=\emptyset$ and $D^{\text {neg }}\left(B^{\prime \prime}, 1 \rightarrow 2\right)=0$.

Let us consider now the influence function $B^{\prime}$ defined by (8). Note that $I_{1 \rightarrow 2}^{\text {neg }}\left(B^{\prime}\right)=$ $\widetilde{I}_{1 \rightarrow 2}$, and $\left(B^{\prime}(\mathbf{i})\right)_{2}=M\left(i_{1}\right)$ for each $\mathbf{i} \in \widetilde{I}_{1 \rightarrow 2}$. Hence,

$$
\int_{I_{1 \rightarrow 2}^{\text {neg }}\left(B^{\prime}\right)}\left[\left|i_{2}-M\left(i_{1}\right)\right|-\left|\left(B^{\prime}(\mathbf{i})\right)_{2}-M\left(i_{1}\right)\right|\right] d \lambda^{2}=\int_{\widetilde{I}_{1 \rightarrow 2}}\left|i_{2}-M\left(i_{1}\right)\right| d \lambda^{2}=\frac{1}{2}
$$

and $D^{\text {neg }}\left(B^{\prime}, 1 \rightarrow 2\right)=1$.

For the influence function $B^{x}$ defined in (10), the negative influence indices will have also a positive value, although smaller than 1 . Let us calculate the negative influence of player 1 on player 2 for $x=\frac{1}{2}$, that is, for the parameter leading to the maximal positive influence index equal to $\frac{7}{16}$.

$$
I_{1 \rightarrow 2}^{n e g}\left(B^{0.5}\right)=\left\{\mathbf{i} \in \widetilde{I}_{1 \rightarrow 2}:\left[\left|i_{2}-i_{1}\right|<\left|\frac{1}{2}-i_{1}\right| \wedge\left|i_{2}-M\left(i_{1}\right)\right|>\left|\frac{1}{2}-M\left(i_{1}\right)\right|\right]\right\}
$$

Figure 9 presents the set $I_{1 \rightarrow 2}^{n e g}\left(B^{0.5}\right)$ which consists of four subsets.

The subsets of $I_{1 \rightarrow 2}^{\text {neg }}\left(B^{0.5}\right)$ are the following:

$$
\begin{gathered}
I_{1 \rightarrow 2}^{\text {negI }}\left(B^{0.5}\right)=\left\{\left(i_{1}, i_{2}\right) \in[0,1]^{2}:\left[0 \leq i_{1} \leq \frac{1}{2} \wedge i_{1} \leq i_{2}<\frac{1}{2}\right]\right\} \\
I_{1 \rightarrow 2}^{\text {negII }}\left(B^{0.5}\right)=\left\{\left(i_{1}, i_{2}\right) \in[0,1]^{2}:\left[0 \leq i_{1} \leq \frac{1}{2} \wedge 2 i_{1}-\frac{1}{2}<i_{2}<i_{1}\right]\right\} \\
I_{1 \rightarrow 2}^{\text {negIII }}\left(B^{0.5}\right)=\left\{\left(i_{1}, i_{2}\right) \in[0,1]^{2}:\left[\frac{1}{2}<i_{1} \leq 1 \wedge i_{1} \leq i_{2}<2 i_{1}-\frac{1}{2}\right]\right\}
\end{gathered}
$$




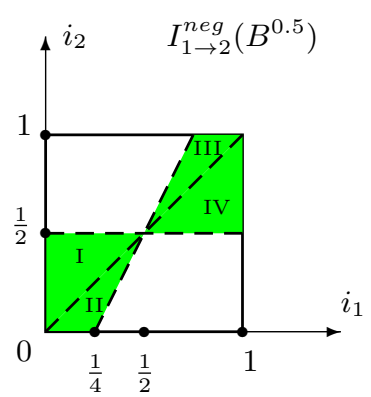

Fig. 9. The set $I_{1 \rightarrow 2}^{\text {neg }}\left(B^{0.5}\right)$ in the 2-player example

$$
I_{1 \rightarrow 2}^{\text {negIV }}\left(B^{0.5}\right)=\left\{\left(i_{1}, i_{2}\right) \in[0,1]^{2}:\left[\frac{1}{2}<i_{1} \leq 1 \wedge \frac{1}{2}<i_{2}<i_{1}\right]\right\} .
$$

The negative influence index of player 1 on player 2 under the influence function $B^{0.5}$ is

$$
\begin{aligned}
D^{\text {neg }}\left(B^{0.5}, 1 \rightarrow 2\right) & =\frac{\int_{I_{1 \rightarrow 2}^{\text {neg }}\left(B^{0.5}\right)}\left[\left|i_{2}-M\left(i_{1}\right)\right|-\left|\frac{1}{2}-M\left(i_{1}\right)\right|\right] d \lambda^{2}}{\int_{\widetilde{I}_{1 \rightarrow 2}}\left|i_{2}-M\left(i_{1}\right)\right| d \lambda^{2}} \\
& =2 \sum_{L \in\{I, I I, I I I, I V\}} \int_{I_{1 \rightarrow 2}^{\text {negL }}\left(B^{0.5}\right)}\left[\left|i_{2}-M\left(i_{1}\right)\right|-\left|\frac{1}{2}-M\left(i_{1}\right)\right|\right] d \lambda^{2} \\
& =2\left[\frac{1}{48}+\frac{1}{16}+\frac{1}{16}+\frac{1}{24}\right]=\frac{3}{8}<\frac{7}{16}=D\left(B^{0.5}, 1 \rightarrow 2\right) .
\end{aligned}
$$

Consequently, under the influence function $B^{0.5}$, the negative influence of player 1 on player 2 is slightly smaller than the positive influence.

The negative influence of player 1 on player 2 for $x=0$ and for $x=1$, that is, for the parameters leading to the minimal positive influence index equal to $\frac{1}{4}$ is equal to

$$
D^{n e g}\left(B^{0}, 1 \rightarrow 2\right)=D^{n e g}\left(B^{1}, 1 \rightarrow 2\right)=\frac{1}{2}>\frac{1}{4}=D\left(B^{0}, 1 \rightarrow 2\right)=D\left(B^{1}, 1 \rightarrow 2\right) .
$$

Hence, under the influence functions $B^{0}$ and $B^{1}$, the negative influence of player 1 on player 2 is greater than the positive influence.

Example 6 For the example presented in Section 3.2 and the function $\widetilde{B}$ defined by (11), similarly as for the function given in $(7)$, we have $I_{13 \rightarrow 2}^{\text {neg[13] }}(\widetilde{B})=\emptyset$ and $D^{\text {neg }}(\widetilde{B}, 13 \rightarrow 2)=0$.

Since the analysis of the negative influence is analogous to the study of the positive influence, in this paper we focus mainly on the positive influence. Consequently, most of the influence functions considered in this paper do not represent any negative influence. We can show that the negative influence index is also equal to zero in the following cases.

Proposition $4 I_{S \rightarrow j}^{\text {neg }}(B)=\emptyset$, and consequently $D^{\text {neg }}(B, S \rightarrow j)=0$, where $B \in \mathcal{B}$, $\emptyset \neq S \subset N$ and $j \in N \backslash S$ are the following:

(i) for each $j \in F_{B}^{\text {per }}(S) \backslash S$, where $F_{B}^{\text {per }}(S)$ is the set of perfect followers of $S$ under $B$;

(ii) for each $B \in \mathcal{B}_{S \rightarrow T}$ (purely influential function of $S$ upon $T$ ); 
(iii) for each majority function $\mathrm{Maj}^{[t]}$, where $n \geq t>\left\lfloor\frac{n}{2}\right\rfloor$, and each $S$ such that $s \geq t$;

(iv) for each guru influence function Gur ${ }^{[\widetilde{k}]}$, and each $S$ such that $\widetilde{k} \in S$;

(v) for the identity function Id.

We omit the proofs here, since they are analogous to the proofs of the corresponding results on the negative influence in the model with a totally ordered set of actions; see Grabisch and Rusinowska (2010b, [31]).

\section{Concluding remarks}

In this paper, we have aimed at defining and investigating the influence model with a continuum of actions and at comparing the results of such a model with the ones obtained in the model with an ordered set of possible actions (and its particular case, the yes-no model). For the continuum case, we have defined and studied the positive and negative influence indices of a coalition on a player, the unanimous influence functions, the set of fixed points under a given influence function, and the linear influence functions. The main difference between the two models lies naturally in the definitions of the influence indices. While in the previous model the influence indices have been defined by sums of some expressions over the particular sets, in the continuum case sums are replaced by integrals. Since some results concerning other tools for analyzing influence (like the set of followers and perfect followers, the purely influential function, the kernel of an influence function, and some properties of the selected unanimous functions) remain the same when switching from the discrete to the continuum case, we did not repeat them in this paper; for the analogous results in the discrete case, see Grabisch and Rusinowska (2010b, [31]).

In the present paper, we have particularly aimed at studying two concepts which we did not consider before, i.e., the fixed points under an influence function, and the linear influence functions. Due to the representation of the linear functions by matrices, such influence functions are very convenient and worth studying. In particular, for the linear functions, being unanimous on the boundaries is equivalent to being unanimous, and is also equivalent to the fact that the matrix is stochastic. According to one of our results, a quick look at a matrix representing an unanimous linear function shows us immediately if there is a positive influence of a coalition on a player and also what the value of the influence index is. Furthermore, we have discussed the convergence of the linear influence functions and the relation between our approach and other linear influence models.

To the best of our knowledge, the influence model with a continuum of actions related to the Hoede-Bakker setting (Hoede and Bakker (1982) [33]) has not been investigated before. Since in real-life situations people frequently have to make a choice of one option from among a continuum of options, we believe that our influence model with a continuum of actions is of importance and contributes significantly to the voting literature.

\section{References}

1. J. Abdou. Neutral veto correspondences with a continuum of alternatives. International Journal of Game Theory, 17:135-164, 1988.

2. C. Asavathiratham. Influence model: a tractable representation of networked Markov chains. PhD thesis, Massachusetts Institute of Technology, Cambridge, MA, 2000. 
3. C. Asavathiratham, S. Roy, B. Lesieutre, and G. Verghese. The influence model. IEEE Control Systems Magazine, 21:52-64, 2001.

4. R. L. Berger. A necessary and sufficient condition for reaching a consensus using DeGroot's method. Journal of the American Statistical Association, 76:415-419, 1981.

5. E. M. Bolger. Power indices for multicandidate voting games. International Journal of Game Theory, 14:175-186, 1986.

6. E. M. Bolger. A value for games with $n$ players and $r$ alternatives. International Journal of Game Theory, 22:319-334, 1993.

7. E. M. Bolger. A consistent value for games with $n$ players and $r$ alternatives. International Journal of Game Theory, 29:93-99, 2000.

8. E. M. Bolger. Characterizations of two power indices for voting games with $r$ alternatives. Social Choice and Welfare, 19:709-721, 2002.

9. P. B. Bonacich. Power and centrality: a family of measures. American Journal of Sociology, 92:1170-1182, 1987.

10. P. B. Bonacich and P. Lloyd. Eigenvector-like measures of centrality for asymmetric relations. Social Networks, 23(3):191-201, 2001.

11. P. Borm, R. van den Brink, and M. Slikker. An iterative procedure for evaluating digraph competitions. Annals of Operations Research, 109:61-75, 2002.

12. M. Braham and F. Steffen. Voting power in games with abstentions. In M.J. Holler, H. Kliemt, D. Schmidtchen, and M.E. Streit, editors, Power and Fairness, pages 333-348. Mohr-Siebeck, 2002.

13. J. Chang and R. Stauber. Group decision-making with a continuum of alternatives and costly participation. Mimeo, 2006.

14. J. Conlisk. Interactive Markov chains. Journal of Mathematical Sociology, 4:157-185, 1976.

15. J. Conlisk. A stability theorem for an interactive Markov chain. Journal of Mathematical Sociology, 6:163$168,1978$.

16. J. Conlisk. Stability and monotonicity for interactive Markov chains. Journal of Mathematical Sociology, $17: 127-143,1992$.

17. R. Cont and M. Löwe. Social distance, heterogeneity and social interactions. Journal of Mathematical Economics, 46:572-590, 2010.

18. M. H. DeGroot. Reaching a consensus. Journal of the American Statistical Association, 69:118-121, 1974.

19. P. DeMarzo, D. Vayanos, and J. Zwiebel. Persuasion bias, social influence, and unidimensional opinions. Quarterly Journal of Economics, 118:909-968, 2003.

20. D. Felsenthal and M. Machover. Ternary voting games. International Journal of Game Theory, 26:335-351, 1997.

21. D. Felsenthal and M. Machover. The Measurement of Voting Power: Theory and Practice, Problems and Paradoxes. London: Edward Elgar Publishers, 1998.

22. D. Felsenthal and M. Machover. Models and reality: the curious case of the absent abstention. In M. J. Holler and G. Owen, editors, Power Indices and Coalition Formation, pages 87-103. Kluwer, Dordrecht, 2001.

23. P. C. Fishburn. The Theory of Social Choice. Princeton University Press, Princeton, 1973.

24. J. Freixas. Banzhaf measures for games with several levels of approval in the input and output. Annals of Operations Research, 137:45-66, 2005.

25. J. Freixas. The Shapley-Shubik power index for games with several levels of approval in the input and output. Decision Support Systems, 39:185-195, 2005.

26. J. Freixas and W. S. Zwicker. Weighted voting, abstention, and multiple levels of approval. Social Choice and Welfare, 21:399-431, 2003.

27. N. E. Friedkin and E. C. Johnsen. Social influence and opinions. Journal of Mathematical Sociology, 15:193206, 1990.

28. N. E. Friedkin and E. C. Johnsen. Social positions in influence networks. Social Networks, 19:209-222, 1997.

29. B. Golub and M. O. Jackson. Naïve learning in social networks and the wisdom of crowds. American Economic Journal: Microeconomics, 2(1):112-149, 2010.

30. M. Grabisch and A. Rusinowska. A model of influence in a social network. Theory and Decision, 69(1):69-96, 2010.

31. M. Grabisch and A. Rusinowska. A model of influence with an ordered set of possible actions. Theory and Decision, 69(4):635-656, 2010.

32. A. Granas and J. Dugundji. Fixed Point Theory. Springer, 2003.

33. C. Hoede and R. Bakker. A theory of decisional power. Journal of Mathematical Sociology, 8:309-322, 1982.

34. C. R. Hsiao and T. E. S. Raghavan. Shapley value for multichoice cooperative games. Games and Economic Behavior, 5:240-256, 1993.

35. W.-H. Huang. Is proximity preservation rational in social choice theory? Social Choice and Welfare, 23:315$332,2004$. 
36. W.-H. Huang. Is a continuum rational social aggregation impossible on continuum spaces? Social Choice and Welfare, 32:635-686, 2009.

37. M. O. Jackson. Social and Economic Networks. Princeton University Press, 2008.

38. L. Katz. A new status index derived from sociometric analysis. Psychometrika, 18:39-43, 1953.

39. U. Krause. A discrete nonlinear and nonautonomous model of consensus formation. In S. Elaydi, G. Ladas, J. Popenda, and J. Rakowski, editors, Communications in Difference Equations. Amsterdam: Gordon and Breach, 2000.

40. J. Lorenz. A stabilization theorem for dynamics of continuous opinions. Physica A, 355:217-223, 2005.

41. J.-H. Steffi Yang. Social network influence and market instability. Journal of Mathematical Economics, 45:257-276, 2009. 